\title{
Self-harm and suicidal ideation during the COVID-19 outbreak in Lebanon: a preliminary study
}

\author{
Ismael Maatouk, Moubadda Assi and Rusi Jaspal \\ Nottingham Trent University, Nottingham, UK
}

\begin{abstract}
Purpose - This cross-sectional study focuses on the social psychological correlates of self-harm and suicidal ideation during the COVID-19 (coronavirus disease 2019) outbreak in Lebanon, which is a country characterized by political and economic instability.

Design/methodology/approach - A convenience sample of 191 Lebanese individuals completed measures of length of being in lockdown, fear of COVID-19, job insecurity, political trust, self-harm and suicidal ideation. Data were analyzed using independent samples $t$-tests and multiple regression.

Findings - Fear of COVID-19 was associated with neither self-harm nor suicidal ideation. Males and people with lower income reported higher suicidal ideation than females and those with higher income, respectively, and religiosity was inversely associated with suicidal ideation. People who reported self-harm generally exhibited lower political trust than those who did not report self-harm.

Research limitations/implications - Beyond the fear of COVID-19, the socioeconomic sequelae of COVID-19 (especially the economic fallout and declining political trust), which are accentuated in already economically and politically unstable societies, may precipitate poor mental health and maladaptive coping strategies in Lebanon. There is a need to ensure adequate access to mental health services to the general population amid the COVID-19 outbreak in Lebanon.

Originality/value - This study provides novel insight into the risk factors for self-harm and suicidal ideation in Lebanon during the COVID-19 pandemic.
\end{abstract}

Keywords COVID-19, Self-harm, Suicidal ideation, Gender, Lebanon

Paper type Research paper

\section{Introduction}

This article presents preliminary data on self-harm and suicidal ideation during the COVID-19 outbreak in Lebanon. COVID-19 is a global pandemic with high rates of morbidity and mortality. As in most countries, the Lebanese prevention strategy has focused on partial or complete national lockdowns and social distancing. Along with its response measures, the pandemic has also been associated with poor mental health outcomes [1]. Fear of COVID-19, being in lockdown and job insecurity are some of the psychological stressors reportedly associated with adverse mental health outcomes during the pandemic [2]. Political and institutional trust can prompt compliance with governmental measures and, along with religiosity, may be protective against poor mental health [3]. It has also been argued that men and women appear to experience the pandemic and the associated lockdown differently and this may be especially true of patriarchal societies, such as Lebanon, in which men and women are expected to conform to strict gender norms [4]. Accordingly, this study focuses on the effects of demographic variables and social psychological variables on two indicators of mental health - self-harm and suicidal ideation - during the pandemic.

(C) Ismael Maatouk, Moubadda Assi and Rusi Jaspal. Published in Journal of Health Research. Published by Emerald Publishing Limited. This article is published under the Creative Commons Attribution (CC BY 4.0) licence. Anyone may reproduce, distribute, translate and create derivative works of this article (for both commercial and non-commercial purposes), subject to full attribution to the original publication and authors. The full terms of this licence may be seen at http://creativecommons.org/licences/by/4.0/ legalcode
Self-harm and suicidal ideation

Received 12 January 2021 Revised 28 February 2021 13 March 2021

Accepted 22 March 2021 
JHR

36,4

706

Societies with existing political and economic instability are likely to be disproportionately affected by the COVID-19 pandemic. During the Lebanese Civil War (1975-1990), hundreds of thousands of people were killed or injured and there was significant damage to the political and economic infrastructure of the country. Years after the end of the Civil War, Lebanon remains a fragile state and has continued to face dramatic economic problems which have worsened with the influx of approximately one million Syrian refugees since 2011 [5]. Long-standing economic and fiscal mismanagement, sectarian politics and refugee crises have rendered Lebanon the third most indebted country in the world. In view of rising unemployment and poverty, Lebanon has experienced increasing political insecurity and citizens have repeatedly protested against the political status quo and demanded change [5].

Lebanon registered its first COVID-19 case on 21 February 2020, and instigated a nationwide lockdown on 15 March 2020 which was extended until July 2020 - the international airport in Beirut, seaports and land entrances were closed as were schools, restaurants and bars [5]. Lockdown measures in Lebanon have coincided with the country facing the worst economic crisis in its history with unprecedented rates of political instability, poverty, job insecurity and devaluation of the local currency [6]. In several studies, fear of COVID-19 has emerged as a key predictor of depression and anxiety [7]. As in other countries, Lebanon has seen a rise in anxiety and post-traumatic stress disorder associated with fear of infection with COVID-19, compounded by job and income insecurities [8]. There has been no published research into self-harm and suicidal ideation during the COVID-19 outbreak in Lebanon. In this study, we examine the impact of social, psychological and economic variables related to the outbreak of self-harm and suicidal ideation in the Lebanese general population.

Maladaptive coping strategies have been reported during the COVID-19 pandemic in countries with scarce and underdeveloped mental health services [6]. In addition to the fear of infection with COVID-19, these studies identified economic and financial crises as predictors of self-harm and suicidal ideation. These findings are consistent with previous studies showing a link between the 2008 economic crisis in Greece and suicidal ideation [9]. It has been claimed that suicide rates in Lebanon are on the rise which has been attributed to financial stress, growing poverty and job insecurity [10]. People within some occupations, such as in healthcare, have been disproportionately affected by poor mental health during the pandemic. Furthermore, studies have reported higher rates of self-harm and suicidal ideation in Lebanon among females and sexual minorities [11]. Generally, treatment-seeking for mental health disorders is less common in Lebanon than in other countries [12].

In Lebanon, lockdown measures have also accentuated existing economic instabilities and threats to the wellbeing of families. During the past year, political and economic instability has led to multiple instances of collective dismissals, as well as salary reductions of up to $70 \%$ in companies, schools and health sector establishments. Although the Lebanese government was able to contain the first wave of COVID-19, it failed to mitigate the social and economic collateral damage associated with the lockdown. Lebanon is a collectivist society with strong religious and cultural norms [11]. Religiosity may function as a coping strategy buffering the effects of poor mental health; it is therefore expected to be negatively correlated with suicidal ideation [11]. Lebanon is also a patriarchal country that favors traditional labor divisions and promotes strictly defined gender roles, with men being assigned the patriarchal role of breadwinner in families and communities. In the absence of integrated mental health services [13] and adaptive coping strategies in Lebanon, such as political and institutional trust, economic instability and job insecurity are psychological stressors that may expose men, in particular, to additional risks to poor mental health manifested in the form of self-harm and suicidal ideation. 


\section{Hypotheses}

The following hypotheses are tested in this study:

H1. Being male and having a lower income will be associated with increased suicidal ideation ideation during the COVID-19 outbreak.

H2. There will be no effect of fear of COVID-19 on self-harm and suicidal ideation.

H3. Political trust will be inversely associated with self-harm during the outbreak.

H4. Religiosity will be inversely associated with suicidal ideation during the outbreak.

\section{Methods \\ Procedure}

The study was publicized through WhatsApp, a cross-platform messaging application. The study link was sent to various nongovernmental organizations and community centers who subsequently used their database of beneficiaries and clients to publicize the study. This was deemed to be an effective means of reaching the Lebanese general population since WhatsApp is a widely used mobile messaging application among most sections of the population. There were two eligibility criteria: being aged 18 years or above and being a resident of Lebanon. Survey responses were collected between March 20 and April 20, 2020. During this period, there was a concomitant general lockdown, which began on March 15, and all people were instructed by the government to stay at home. In order to generate data for this study, which took place during the national lockdown, a convenience sampling approach (using an online data collection method) was deemed to be most appropriate during this period.

\section{Participants}

A convenience sample of 191 individuals participated in the survey. Participants were aged between 17 and $66(M=29.87, S D=8.06)$. There were $120(62.8 \%)$ males and $71(37.2 \%)$ females, Table 1.

\section{Measures}

The original English-language measures were translated from English into Arabic and then back-translated into English by the first author. They were cross-checked by the second author. Participants were given the option to complete the survey in either English or Arabic.

Demographic questions included age; gender and current monthly income. Length of being in lockdown was measured using a 4-point scale $(1=$ less than one week, $4=$ more than 4 weeks). Suicidal ideation was assessed using the 4-item Suicide Behaviors QuestionnaireRevised (SBQ-R) [14]. Example item: "Have you ever thought about or attempted to kill yourself?". Possible scores were 4 to 23. A sum score provided an overall score of suicidal ideation - the higher the score, the higher the level of suicidal ideation $(\alpha=0.85)$. Self-harm was measured using the following item from the Adult Psychiatric Morbidity Survey [15]: "Have you ever deliberately harmed yourself in any way but not with the intention of killing yourself?" ("yes" or "no"). Fear of COVID-19 was assessed using the 10-item Fear of COVID-19 Scale (FCV-19S) Questionnaire[16]. Example item: "I am most afraid of COVID-19". Possible scores were 10 to 50. A sum score provided an overall score of fear of COVID-19 - the higher the score, the higher the level of fear $(\alpha=0.81)$. Job insecurity was assessed using the 4item Job Insecurity Scale Questionnaire [17]. Example item: "I feel insecure about the future of my job”. Specific items were reverse scored. Possible scores were 4 to 20. A sum score provided an overall score of job insecurity - the higher the score, the higher the level of 


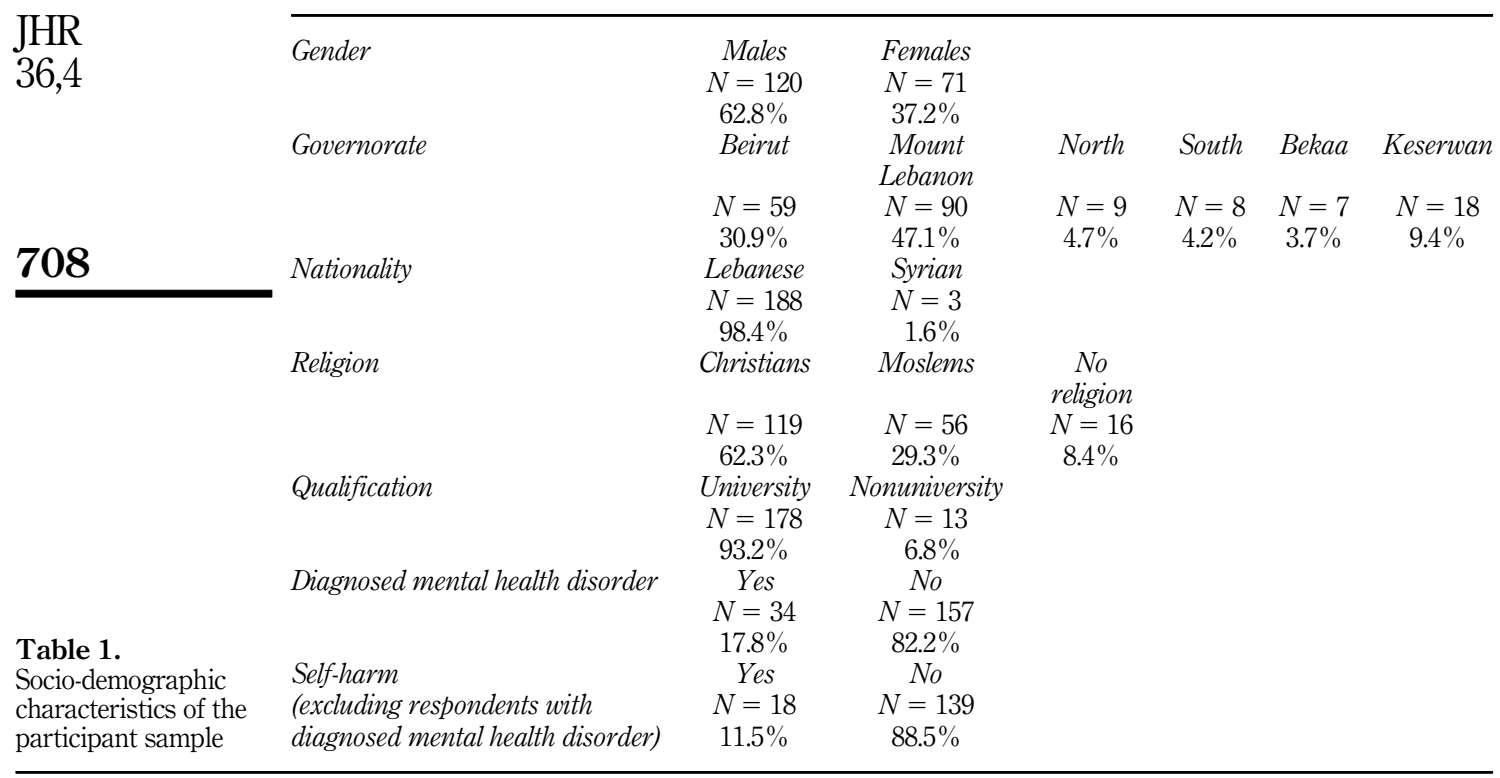

insecurity $(\alpha=0.86$ ). Political trust was assessed using the 12-item Political Trust Questionnaire [18]. Example item: "Politicians generally have good intentions". Possible scores were 0 to 48. A sum score provided an overall score of political trust - the higher the score, the higher the level of trust $(\alpha=0.85)$.

\section{Results}

Exclusion of participants with diagnosed mental health disorders

The sample included 34 participants $(17.8 \%)$ with diagnosed mental health disorders. Independent samples $t$-tests showed statistically significant differences for suicidal ideation $[t(36.8)=3.57, p<.005$; Cohen's $d=1.09 ; 95 \%$ CIs $(1.24953,4.52229)]$ between respondents who had a diagnosed mental health disorder and those who did not. Generally, those who have a diagnosed mental health disorder showed higher levels of suicidal ideation $(M=6.14, S D=4.58$ vs $M=3.26, \mathrm{Sd}=2.34$ ) when compared to those who did not. In order to focus on suicidal ideation in individuals with no prior mental health diagnosis (i.e. nonclinical participants), people with a diagnosed mental health disorder were excluded from further analyses.

\section{Descriptive statistics}

Table 2 provides a full summary of the descriptive statistics concerning the key variables of interest. On average, people expressed high fear of COVID-19, moderate levels of religiosity and low levels of political trust.

\section{Socioeconomic differences for suicidal ideation and self-harm}

Independent samples $t$-tests showed a statistically significant difference in suicidal ideation for males and females $[t(137.6)=-4.557, p<0.001$; Cohen's $d=0.66$; 95\% CIs $(0.76618$, $1.94078)]$, as well as high and low income levels $[t(108.1)=2.487, p<0.05$; Cohen's $d=0.41$; $95 \%$ CIs $(0.19962,1.76745)]$. Generally, levels of suicidal ideation were higher for males when 
compared to females $(M=3.73, \mathrm{Sd}=2.71$ vs $M=2.38, \mathrm{Sd}=0.93)$ and for the low-income group when compared to the high-income group $(M=3.84, \mathrm{Sd}=2.71$ vs $M=2.86, \mathrm{Sd}=1.98)$.

Further independent samples $t$-tests showed a statistically significant difference in length of being in lockdown $[t(30.7)=2.348, p<0.05$; Cohen's $d=0.48$; 95\% CIs $(0.046,0.66)]$ between those who reported self-harm and those who did not. Generally, length of being in lockdown was higher for those who reported self-harm when compared to those who did not $(M=3.78, \mathrm{Sd}=0.55$ vs $M=3.42, \mathrm{Sd}=0.91)$.

Differences between those who engaged in self-harm and those who did not

Independent samples $t$-tests showed a statistically significant difference between those who engaged in self-harm and those who did not for political trust $[t(155)=-2.177, p<0.05$; Cohen's $d=; 95 \%$ CIs $(-7.26524,-0.35347)]$. Generally, political trust was higher among those who did not self-harm when compared to those who did self-harm $(M=9.31, \mathrm{Sd}=6.91$ vs $M=5.5, \mathrm{Sd}=7.54$ ).

Further independent samples $t$-tests showed no statistically significant difference in fear of COVID-19 between those who did and those who did not self-harm $(\phi>0.05)$.

\section{Correlations between key variables}

The results indicated positive correlations between suicidal ideation and job insecurity. In contrast, suicidal ideation was negatively correlated with religiosity. There was no significant correlation between suicidal ideation and fear of COVID-19, Table 3.

\section{Multiple regression model predicting suicidal ideation}

A multiple stepwise regression was conducted to examine which variables predicted the variance of suicidal ideation. The continuous variables of religiosity and job insecurity as well as the dichotomous variables of gender $($ female $=0$, male $=1)$ and income $(0=$ low, $1=$ high $)$ were inserted as predictors, and suicidal ideation was inserted as the dependent variable.

\begin{tabular}{lrrrr}
\hline & Mean & Sd & Minimum & Maximum \\
\hline Age & 29.64 & 7.72 & 18 & 66 \\
Religiosity & 13.11 & 3.78 & 5 & 20 \\
Length of being in lockdown & 3.46 & .88 & 1 & 4 \\
Suicidal ideation & 5.26 & 2.34 & 4 & 15 \\
Fear of COVID-19 & 22.28 & 5.25 & 10 & 36 \\
Job insecurity & 14.19 & 3.69 & 4 & 20 \\
Political trust & 8.87 & 7.06 & 0 & 26 \\
\hline
\end{tabular}

Table 2.

Descriptive statistics for the key variables of this study

\begin{tabular}{lccrrrrr}
\hline & 1 & 2 & 3 & 4 & 5 & 6 & 7 \\
\hline 1. Age & & 0.07 & -0.08 & $-0.18^{*}$ & 0.07 & -0.07 & -0.11 \\
2. Religiosity & 0.07 & & 0.05 & -0.08 & $0.19^{*}$ & 0.13 & $-0.25^{* *}$ \\
3. Length of being in lockdown & -0.08 & 0.05 & & 0.03 & -0.14 & 0.09 & 0.06 \\
4. Job insecurity & $-0.18^{*}$ & -0.08 & 0.03 & & $-0.21^{* *}$ & 0.14 & $0.19^{*}$ \\
5. Political trust & 0.07 & $0.19^{*}$ & -0.14 & $-0.21^{* *}$ & & -0.15 & -0.14 \\
6. Fear of COVID-19 & -0.07 & 0.13 & 0.09 & 0.14 & -0.15 & & 0.09 \\
7. Suicidal ideation & -0.11 & $-0.25^{* *}$ & 0.06 & $0.19^{*}$ & -0.14 & 0.09 &
\end{tabular}

Note(s): ${ }^{*} p<0.050 ; * * p<0.005$ 
JHR

36,4

710

Gender entered in Step 1 and explained 7\% of the variance in suicidal ideation. At step 2, gender and income explained $10.3 \%$ of the variance in suicidal ideation. $R$-square change was 0.035 and $F$-change was $6.078(p=0.015)$. At step 3 , gender, income and religiosity explained $12.9 \%$ of the variance in suicidal ideation. $R$-square change was 0.03 and $F$-change was 6.121 $(\phi=0.014)$. The regression model was statistically significant for suicidal ideation $[F(3$, $\left.153)=8.686, p<0.01 ; R^{2}=0.129\right]$. Of all predictors, gender (being male) with a $\beta=0.221$ S.E. $=0.097,95 \%$ CIs $(0.079,0.462)(t=2.792, p<0.01)$ was the most powerful, followed by income with a $\beta=-0.200$ S.E. $=0.012,95 \%$ CIs $(-0.056,-0.008)(t=-2.668, p<0.01)$ and religiosity with a $\beta=-0.196$ S.E. $=0.061,95 \%$ CIs $(-0.274,-0.031)(t=-2.474, p<0.05)$ and had significant effects on the variance of suicidal ideation. The variable of job insecurity was excluded from the model in the 3 steps.

\section{Discussion}

This preliminary study provides insight into self-harm and suicidal ideation in the Lebanese population during the COVID-19 outbreak. Overall, our findings support the four hypotheses. Being male and having a low income was associated with higher suicidal ideation. Fear of COVID-19 was not associated with self-harm or suicidal ideation, while those who engaged in self-harm reported lower political trust that those who did not. Religiosity was negatively correlated with suicidal ideation.

It is recognized that the design of our study does not allow us to attribute unequivocally the findings to the outbreak of COVID-19 in Lebanon since we did not collect data prior to the outbreak which could serve as a baseline. However, it is clear that the pandemic has accentuated some of the issues (e.g. economic deprivation, inability to engage with community and religious groups) which, in our study, are indeed associated with increased suicidal ideation. Moreover, the COVID-19 pandemic may have differential implications for health in accordance with one's gender in particular cultural contexts. Our data from Lebanon suggest that males are at higher risk of suicidal ideation compared to females. This supports hypothesis 1 . Collectivist patriarchal societies such as that of Lebanon tend to prescribe gender roles based on a social obligation for males to provide for their families and to ensure financial security. Although Lebanon is more liberal than neighboring Arab countries in relation to gender, males are still regarded as essential breadwinners in Lebanese society [4]. During these insecure times in Lebanon characterized by job insecurity and financial instability, this culturally-assigned role may be generating additional psychological stress among males. In the absence of adaptive coping strategies, such as community and religious engagement, men who are facing distress associated with COVID-19 may be at especially high risk of suicidal ideation.

Depressive symptomatology (depression and anxiety) during the pandemic has been attributed to fear of infection with COVID-19 globally and in Lebanon [8]. On the other hand, maladaptive coping strategies (self-harm and suicidal ideation) have mostly been reported in societies that are facing major economic and political fallouts due to the pandemic [9]. We did not find an association in our sample between either self-harm or suicidal ideation and fear of COVID-19, which supported hypothesis 2 . However, in support of hypotheses 1 and 3 , we did find a significant association between income level and suicidal ideation and between political trust and self-harm. Consistent with work showing the protective functions performed by religiosity [11], we found that religiosity was negatively associated with suicidal ideation, which was consistent with hypothesis 4 . This suggests a strong socioeconomic component to poor mental health following the COVID-19 outbreak in Lebanon. The economic crisis in Lebanon started long before the pandemic, but has been compounded by lockdown measures. In fact, several cases of suicide were reported as a result of financial insecurities prior to the pandemic [8]. Whereas political and institutional trust can be viewed as an adaptive coping 
strategy in the face of major crises [3], this may not be accessible to many parts of the Lebanese society amid intractable geopolitical tensions and divisions in the country [6].

Therefore, the socio-economic sequelae of COVID-19 (especially the economic fallout), which are accentuated in economically and politically unstable societies, may be leading to poor mental health and maladaptive coping strategies in Lebanon. Furthermore, mental health services in Lebanon are still in their formative stages and are not integrated in a package of services at the national level $[6,13]$. Whereas early management of depression and other mental health disorders is the most important suicide-prevention strategy [10], the absence of effective mental health services at the national level in Lebanon could eventually lead to maladaptive coping strategies [6].

This preliminary study has some limitations, which should be addressed in future work. First, this cross-sectional study provides only an empirical snapshot of suicidal ideation and self-harm at a given point in time but does not allow us to determine causal relationships between the key variables. Future research using experimental, longitudinal and crosssequential designs would be valuable. Furthermore, given that no data were collected before the outbreak of COVID-19 in Lebanon, it is unclear whether the findings can be attributed to COVID-19 in particular or to broader malaise in the country due to pre-existing political and economic issues. Further research will help delineate the impact of COVID-19, in particular. Second, the convenience sample was recruited via nongovernmental organizations and community centers and, thus, it is difficult to generalize the results to the Lebanese population. Though challenging during the pandemic, it would be advantageous to attempt to recruit more representative samples of the Lebanese population. Third, the vast majority of our sample consisted of Lebanese citizens, and migrant groups, such as Syrians and Palestinians, were clearly underrepresented. It will be important for future research to include sufficient samples of migrant groups in order to provide a more comprehensive understanding of how COVID-19 may be affecting people in Lebanon.

In countries with political and economic instability, such as Lebanon, adaptive coping strategies may be less readily available which could lead to an increased mental health burden in response to the COVID-19 outbreak. There is a need to ensure adequate access to mental health services in the general population in Lebanon. Amid the need for social distancing as well as prevention measures during the COVID-19 pandemic, a tele-mental health approach may help address the mental health burden in the general population and, particularly, in vulnerable communities across Lebanon. Moreover, in our study, religiosity and suicidal ideation were negatively correlated. It may be advantageous to build resilience and problem-solving skills that are deemed to be compatible with religion and spirituality, in order to promote effective coping during the pandemic. This approach has been shown to be effective in relieving stress in Italy, for instance [19]. Furthermore, healthcare workers who have been especially vulnerable to poor mental health during the pandemic may benefit from approaches that can promote sleep quality and access to psychological support, as demonstrated in recent research conducted in Italy [20].

\section{Conclusion}

This preliminary study provides some insight into self-harm and suicidal ideation in a Lebanese sample during the initial phase of the COVID-19 pandemic. Being male and having a low income appear to be risk factors for suicidal ideation, while political trust is inversely associated with self-harm, and religiosity is inversely associated with suicidal ideation. In societies characterized by political and economic instability and in which religion and community are central tenets of identity, the COVID-19 pandemic may result in significant mental health challenges at a population level. It would be advantageous to explore novel and innovative approaches to enhancing mental health in the general population, such as the tele-
Self-harm and suicidal ideation 
JHR

36,4

health approach. This may increase both the reach and effectiveness of mental health support in Lebanon.

Conflict of Interest: None

\section{References}

1. Lopes B, Jaspal R. Understanding the mental health burden of COVID-19 in the United Kingdom. Psychol Trauma. 2020; 12(5): 465-7. doi: 10.1037/tra0000632.

2. Satici B, Saricali M, Satici SA, Griffiths MD. Intolerance of uncertainty and mental wellbeing: serial mediation by rumination and fear of COVID-19. Int J Ment Health Addict. 2020: 1-12. doi: 10. 1007/s11469-020-00305-0.

3. Cheung CK, Tse JW. Institutional trust as a determinant of anxiety during the SARS crisis in Hong Kong. Soc Work Public Health. 2008; 23(5): 41-54. doi: 10.1080/19371910802053224.

4. Dirani K. Exploring socio-cultural factors that influence HRD practices in Lebanon. Hum Resour Dev Int. 2006; 9(1): 85-98. doi: 10.1080/13678860500523270.

5. Abouzeid M, Habib RR, Jabbour S, Mokdad AH, Nuwayhid I. Lebanon's humanitarian crisis escalates after the Beirut blast. Lancet. 2020; 396(10260): 1380-2. doi: 10.1016/S0140-6736(20) 31908-5.

6. Jaspal R, Assi M, Maatouk I. Potential impact of the COVID-19 pandemic on mental health outcomes in societies with economic and political instability: case of Lebanon. Ment Health Rev J. 2020; 25(3): 215-9. doi: 10.1108/mhrj-05-2020-0027.

7. Fitzpatrick KM, Harris C, Drawve G. Fear of COVID-19 and the mental health consequences in America. Psychol Trauma. 2020; 12(S1): S17-21. doi: 10.1037/tra0000924.

8. Khoury B, El-Khoury J, Ammar J. Psychological needs and response during the COVID-19 pandemic in Lebanon. Psychol Trauma. 2020; 12(5): 497-8. doi: 10.1037/tra0000757.

9. Economou M, Madianos M, Peppou LE, Theleritis C, Patelakis A, Stefanis C. Suicidal ideation and reported suicide attempts in Greece during the economic crisis. World Psychiatr.. 2013; 12(1): 53-9. doi: 10.1002/wps.20016.

10. Bou Khalil R. The psychological wellbeing of the Lebanese society lies between incremental suicide rates and financial stress. Asian J Psychiatr. 2019; 42: 85-6. doi: 10.1016/j.ajp.2019.04.002.

11. Assi M, Maatouk I, Jaspal R. Psychological distress and self-harm in a religiously diverse sample of Lebanese students. Ment Health Relig Cult. 2020; 23(7): 591-605. doi: 10.1080/13674676.2020. 1788524 .

12. Maalouf FT, Alrojolah L, Ghandour L, Afifi R, Dirani LA, Barrett P, et al. Building emotional resilience in youth in Lebanon: a school-based randomized controlled trial of the FRIENDS intervention. Prev Sci. 2020; 21(5): 650-60. doi: 10.1007/s11121-020-01123-5.

13. El Chammay R, Roberts B. Using COVID-19 responses to help strengthen the mental health system in Lebanon. Psychol Trauma. 2020; 12(S1): S281-3. doi: 10.1037/tra0000732.

14. Osman A, Bagge CL, Gutierrez PM, Konick LC, Kopper BA, Barrios FX. The Suicidal Behaviors Questionnaire-Revised (SBQ-R): validation with clinical and nonclinical samples. Assessment. 2001; 8(4): 443-54. doi: 10.1177/107319110100800409.

15. McManus S, Bebbington P, Jenkins R, Brugha T. Mental Health and Wellbeing in England: adult psychiatric morbidity survey 2014. Leeds: NHS digital; 2016.

16. Ahorsu DK, Lin CY, Imani V, Saffari M, Griffiths MD, Pakpour AH. The fear of COVID-19 Scale: development and initial validation. Int J Ment Health Addict. 2020: 1-9. doi: 10.1007/s11469-02000270-8.

17. Vander Elst T, De Witte H, De Cuyper N. The job insecurity scale: a psychometric evaluation across five European countries. Eur J Work Organ Psychol. 2014; 23(3): 364-80. doi: 10.1080/ 1359432X.2012.745989. 
18. Mutz DC, Reeves B. The new videomalaise: effects of televised incivility on political trust. Am Polit Sci Rev. 2005; 99(1): 1-15. doi: 10.1017/S0003055405051452.

19. Chirico F, Nucera G. An Italian experience of spirituality from the coronavirus pandemic. J Relig Health. 2020; 59(5): 2193-5. doi: 10.1007/s10943-020-01036-1.

Self-harm and suicidal ideation

20. Chirico F, Nucera G, Magnavita N. Protecting the mental health of healthcare workers during the COVID-19 emergency. BJPsych Int. 2021; 18(1): e1.1-2. doi: 10.1192/bji.2020.39.

\section{Corresponding author}

Rusi Jaspal can be contacted at: rusi.jaspal@cantab.net 